\title{
Effect of suprathermal particles on the quiet Sun radio emission ${ }^{\star}$
}

\author{
C. Chiuderi and F. Chiuderi Drago
}

Dipartimento di Astronomia e Scienza dello Spazio, largo E. Fermi 2, 50125, Firenze, Italy

Received 2 December 2003 / Accepted 31 March 2004

\begin{abstract}
The bremsstrahlung emissivity and absorption coefficient in the radiofrequency range are derived under the assumption that the electron population is not purely thermal, but presents a tail of high energy particles. This population is approximated by a two-component Maxwellian distribution and by the kappa-functions of different (integer) index. It is shown that, if the temperature ratio of the two Maxwellians is larger than 10, the absorption coefficient and the effective temperature (the quantities entering the radio transfer equation) depend only on the fraction $R$ of particles in the highest temperature Maxwellian. In the case of kappa-functions the above quantities depend on the index $n$ of the functions.

The microwave radio spectrum is computed for different values of $R$ and for $3 \leq n \leq 6$, finding, in all cases, brightness temperatures lower than those computed with a pure thermal distribution. This could explain some inconsistencies found between radio and EUV observations.
\end{abstract}

Key words. radiation mechanisms: non-thermal - Sun: transition region - Sun: corona - Sun: radio radiation

\section{Introduction}

The upper layers of the solar atmosphere, transition region (TR) and corona, can be investigated by means of the radiation emitted in the X-UV and radio domain. The UV and EUV line intensities emitted in the TR depend strongly on temperature and are proportional to the differential emission measure: $\operatorname{DEM}(T)=N_{\mathrm{e}}^{2} \mathrm{~d} h / \mathrm{d} T$. Those emitted in the corona, where $T \sim$ constant, are instead proportional to the emission measure $E M=\int N_{\mathrm{e}}^{2} \mathrm{~d} h$. It turns out that in the microwave range, where the refraction index can be safely assumed equal to unity, the resulting brightness temperature $T_{\mathrm{b}}$ depends precisely on the same parameters. However, the radio emission computed using models of the solar atmosphere inferred from EUV observations do not agree with the observations (see, for instance, Zirin et al. 1981; Noci 2003).

Landi \& Chiuderi Drago (2003) (hereafter referred to as Paper I) have recently shown that, using a composite TR model of the cell center that excludes the DEM derived from UV lines at low temperature, a decent agreement with the observed quiet sun $T_{\mathrm{b}}$ can be achieved. However, it is clear that any contribution of the network to the computed $T_{\mathrm{b}}$ will spoil again this agreement.

All this seems to indicate that a deeper reason for inconsistency exists in the calculation of the microwave spectra, which appear to be systematically in excess of the observations.

To investigate an alternative possibility of decreasing the computed radio brightness temperature $\left(T_{\mathrm{b}}\right)$, in this paper we

\footnotetext{
Send offprint requests to: F. Chiuderi Drago,

e-mail: Chiuderi@arcetri.astro.it

* Appendix is only available in electronic form at http://www. edpsciences.org
}

will compute the quiet sun microwave spectrum under the assumption that a small tail of suprathermal electrons is present in the upper part of the solar atmosphere. The possible presence of such high energy tail has attracted a considerable attention in the last few years. The origin of stationary non-Maxwellian electron distribution functions could be traced in some acceleration process effective in in the chromosphere or in the lower transition region (Viñas et al. 2000) or in the transport of highenergy electrons from the upper transition region and corona (Ljepojevic \& Burgess 1990). In this paper we shall not dwell on the question of the generation and maintenance of this type of distribution, but simply assume their existence and trace the observable consequences of this assumption in the radio range.

The tail of suprathermal electrons will be modelled either assuming an electron distribution obtained by adding two Maxwellians at different temperatures or by a so-called kappafunction with an integer index $n: 3 \leq n \leq 6$. Since we want to compare our results with those obtained by assuming a Maxwellian distribution, the values of the parameters of the modified distributions will be always chosen to ensure that the density and the mean energy of the distributions equal those of the reference Maxwellian.

The assumption that the electron distribution in the solar and stellar coronae and in the thin transition region between the corona and the "visible underlying photospheric/chromospheric surface" could follow a kappa-function was first put forward by Scudder (1992). With this assumption he was able to explain several observations concerning the TR, the corona and the solar wind such as the steep positive gradient in the TR, the Doppler width of UV lines, the critical point location and the asymptotic velocity of the solar wind. 
This assumption has been applied later on by several authors to account for the electron distribution observed by Ulysses (Maksimovic et al. 1997), the heat flux in the solar corona (Landi \& Pantellini 2001; Sittler 2002) and in several solar wind models (Maksimovic et al. 1997; Sheldon et al. 2001).

The kappa-functions have also been used in an attempt to calculate the gyrosynchrotron emission at radiowavelengths in non-flaring active regions, but the agreement with the observations has been found unsatisfactory.

The calculation of the emission and absorption coefficients for a two-component Maxwellian and for kappa-function distributions will be presented in the next section. In Sect. 3 we will compute the microwave spectra, obtained by two-component Maxwellian distributions with different ratios of temperature and number of particles and by a kappa-function with different index $n$. The results will be compared with the Zirin et al. (1991) and Borovik et al. (1992) observations in Sect. 4. A summary and the conclusions are given in Sect. 5.

\section{Emission and absorption from electrons with a generic distribution function.}

The general theory of the emission and absorption of electromagnetic waves from an ensemble of electrons with a generic distribution function has been developed long ago (see e.g., Wild et al. 1963; Zheleznyakov 1970). We simply recall here the basic equations, following the treatment by Wild et al. (1963).

If $p$ and $E=p^{2} / 2 m$ are respectively the momentum and energy of a single electron, the momentum distribution function, $f(p)$, can be defined by means of the integral:

$N_{\mathrm{e}}=\int_{0}^{\infty} f(p) 4 \pi p^{2} \mathrm{~d} p$,

where $N_{\mathrm{e}}$ is the number density of electrons in ordinary space. By transforming the momentum integration into an energy integration, the above normalization condition becomes:

$N_{\mathrm{e}}=\int_{0}^{\infty} F(E) E^{1 / 2} \mathrm{~d} E$,

where the definition of $F(E)$ includes all the constant factors coming from the above variable transformation. In case of non-relativistic particles:

$F(E)=4 \pi\left(2 m^{3}\right)^{1 / 2} f(p)$.

With these definitions the Maxwellian distribution function can be written as:

$F_{0}(E)=\frac{2}{\sqrt{\pi}} N_{\mathrm{e}}\left(k_{\mathrm{B}} T\right)^{-3 / 2} \mathrm{e}^{-E / k_{\mathrm{B}} T}$,

where $k_{\mathrm{B}}$ is the Boltzmann constant. The temperature, $T$, of a Maxwellian distribution is related to the average of the single particle energy over the distribution function:

$\langle E\rangle=\frac{1}{N_{\mathrm{e}}} \int_{0}^{\infty} E^{3 / 2} F(E) \mathrm{d} E=\frac{3}{2} k_{\mathrm{B}} T$.
In this paper the "temperature" will be always defined by the above formula, irrespective of the form of the distribution function.

If $\epsilon_{\nu}(E)$ is the free-free radio emission in one polarization per unit time and unit solid angle from a single electron of energy $E$ in a plasma with an ion density $N_{i}$, the total emissivity and the absorption coefficient of an ensemble of electrons distributed according to a generic $F(E)$ are given by (Wild et al. 1963):

$j(v)=2 \int_{0}^{\infty} \epsilon_{v}(E) F(E) E^{1 / 2} \mathrm{~d} E$

and

$k(v)=-\frac{c^{2}}{v^{2}} \int_{0}^{\infty} \frac{\mathrm{d} F(E)}{\mathrm{d} E} \epsilon_{v}(E) E^{1 / 2} \mathrm{~d} E$.

In the above formulas we have assumed the refraction index of the medium equal to unity, as appropriate to the microwave range of frequencies.

The source function is defined as the ratio between the emissivity and the absorption coefficient:

$S(v)=j(v) / k(v)$

and an effective temperature, $T_{\text {eff }}$, can always be defined using the equation:

$S(v)=\frac{2 k_{\mathrm{B}} T_{\mathrm{eff}} v^{2}}{c^{2}}$

The single particle free-free radioemission in one polarization and per unit time is given by (see e.g., Scheuer 1960; Oster 1961):

$\epsilon_{v}(E)=C \frac{N_{i}}{E^{1 / 2}}(\ln g(E, v)-\gamma)$,

where the constant $\mathrm{C}$ is given by:

$C=\frac{2^{3 / 2} Z^{2} e^{6}}{3 m^{3 / 2} c^{3}}=1.56 \times 10^{-47}$,

$g(E, v)=\frac{(2 E)^{3 / 2}}{\pi Z e^{2} m^{1 / 2} v}$

and $\gamma$ is the Euler constant,

$\gamma=\int_{0}^{\infty} \ln (x) \mathrm{e}^{-x} \mathrm{~d} x=0.577 \ldots$

If $F(E)$ is a Maxwellian at temperature $T$ the integrals appearing in Eqs. (2) and (3) can be computed explicitly (see Scheuer 1960), giving:

$j_{0}(v)=\frac{2 C}{\sqrt{\left(\pi k_{\mathrm{B}}\right)}} \frac{N_{i} N_{\mathrm{e}}}{T^{1 / 2}} \Lambda$,

$k_{0}(v)=\frac{2 C}{\left.\sqrt{(} \pi k_{\mathrm{B}}\right)} \frac{c^{2} / v^{2}}{2 k_{\mathrm{B}}} \frac{N_{i} N_{\mathrm{e}}}{T^{3 / 2}} \Lambda$,

where

$\Lambda=\ln \left(\alpha T^{3} / \nu^{2}\right)-5 \gamma$, 
with

$\alpha=\frac{8 k_{\mathrm{B}}^{3}}{\pi^{2} Z^{2} e^{4} m}=4.41 \times 10^{16}$ (cgs units).

The effective temperature in this case is equal to the temperature, as expected in thermal equilibrium.

We consider now the case of a two-component Maxwellian, namely a distribution function obtained by adding two Maxwellians at different temperatures $T_{0}$ and $T_{1}$ (with $T_{0}<T_{1}$ ) and different densities $N_{0}$ and $N_{1}$ (with $N_{0}>N_{1}$ ). Such a distribution could be representative of a system made up of a relatively large number of "cold" electrons to which a small amount of "hot" electrons is added. A certain care should be applied when choosing the values of the parameters $T_{0}, T_{1}, N_{0}$ and $N_{1}$ to avoid the occurrence of instabilities of the beamplasma type. Although the degree of realism of such a description is questionable, the two-component Maxwellian appears to be a practical parametrization of situations in which a high-energy tail is superimposed on an otherwise thermal distribution.

The results for a two-component Maxwellian follow directly from those of a Maxwellian. Introducing

$R=\frac{N_{1}}{\left(N_{0}+N_{1}\right)}=N_{1} / N_{\mathrm{e}}$,

the ratio of the density of the hot electrons to the total density, $N_{\text {e }}$, we can write:

$j(v)=j_{0}(v) \times(1-R)\left(1+\frac{R}{1-R} \frac{\Lambda_{1}}{\Lambda_{0}}\left(\frac{T_{0}}{T_{1}}\right)^{0.5}\right)$,

$k(v)=k_{0}(v) \times(1-R)\left(1+\frac{R}{1-R} \frac{\Lambda_{1}}{\Lambda_{0}}\left(\frac{T_{0}}{T_{1}}\right)^{1.5}\right)$.

where $\Lambda_{0,1}$ are defined as $\Lambda$ in Eq. (5), but they are evaluated at $T=T_{0}$ and $T=T_{1}$, respectively.

The effective temperature turns out to be:

$T_{\text {eff }}=T_{0} \times \frac{1+\frac{\Lambda_{1}}{\Lambda_{0}} \frac{R}{(1-R)}\left(\frac{T_{0}}{T_{1}}\right)^{0.5}}{1+\frac{\Lambda_{1}}{\Lambda_{0}} \frac{R}{1-R}\left(\frac{T_{0}}{T_{1}}\right)^{1.5}}$.

Comparing this result with that referring to a Maxwellian distribution at $T_{0}$, it is easy to see that $T_{\text {eff }}$ is always greater than $T_{0}$.

We finally turn to the case of a distribution function described by a kappa-function, that we write as:

$F_{\kappa}(E)=\frac{2}{\sqrt{\pi}} N_{\mathrm{e}}\left(k_{\mathrm{B}} T\right)^{-3 / 2} H_{\kappa} G_{\kappa}(E)$,

where

$H_{\kappa}=\frac{(\kappa-3 / 2)^{-3 / 2} \Gamma(\kappa+1)}{\Gamma(\kappa-1 / 2)}$

and

$G_{\kappa}(E)=\left(1+(\kappa-3 / 2)^{-1} \frac{E}{k_{\mathrm{B}} T}\right)^{-(\kappa+1)}$.
In the above equations $\Gamma(z)$ is the Euler function and $T$ is the temperature defined as in Eq. (1). The kappa-functions are defined for all real values of $\kappa>3 / 2$ and converge to the Maxwellian when $\kappa$ tends to infinity. In the following only integer values for $\kappa$ will be considered, so that $\kappa=n \geq 2$.

The integrals entering Eqs. (2) and (3) can be evaluated explicitly also when $F(E)$ is a kappa-function. Some details are presented in the Appendix, to which we refer the interested reader. Defining $j_{n}$ as the emissivity computed for a kappafunction with $\kappa=n$ and $k_{n}$ as the corresponding absorption coefficient, we obtain:

$j_{n}(v)=\frac{2 C}{\sqrt{\pi k_{\mathrm{B}}}} \frac{N_{i} N_{\mathrm{e}}}{T^{1 / 2}} \Lambda \frac{n-3 / 2}{n} H_{n}\left(1+3 \frac{\left(\Omega+\frac{1}{n}\right)}{\Lambda}\right)$

and

$k_{n}(v)=\frac{2 C}{\sqrt{\pi k_{\mathrm{B}}}} \frac{c^{2} / v^{2}}{2 k_{\mathrm{B}} T} \frac{N_{i} N_{\mathrm{e}}}{T^{3 / 2}} \Lambda H_{n}\left(1+3 \frac{\Omega}{\Lambda}\right)$,

where $\Lambda$ is defined as in Eq. (5) and

$\Omega=\gamma+\ln (n-3 / 2)-\sum_{k=1}^{n} 1 / k$

Substituting into the above Equations the expression of $j_{0}$ and $k_{0}$,we obtain:

$j_{n}(v)=j_{0}(v) \times \frac{n-3 / 2}{n} H_{n}\left(1+3 \frac{(\Omega+1 / n)}{\Lambda}\right)$

and

$k_{n}(v)=k_{0}(v) \times H_{n}\left(1+3 \frac{\Omega}{\Lambda}\right)$.

From these expressions we can evaluate the source function and the effective temperature $T_{\text {eff. }}$ The latter turns out to be:

$$
\begin{aligned}
T_{\text {eff }} & =T \frac{n-3 / 2}{n} \frac{\Lambda+3(\Omega+1 / n)}{\Lambda+3 \Omega} \\
& =T \frac{n-3 / 2}{n}\left(1+\frac{3 / n}{\Lambda+3 \Omega}\right) .
\end{aligned}
$$

It can be verified (see Appendix) that $\lim _{n \rightarrow \infty} H_{n}=1$ and $\lim _{n \rightarrow \infty} \Omega=0$, so that $T_{\text {eff }}$ converges to $T$ when $n \rightarrow \infty$.

\section{Comparison with observations}

The two quantities entering the radio transfer equation:

$T_{\mathrm{b}}(v)=\int T_{\text {eff }} \mathrm{e}^{-\tau_{v}} \mathrm{~d} \tau_{v}$

are the effective temperature $T_{\text {eff }}$ and the absorption coefficient $k(v)$, related to the optical depth, $\mathrm{d} \tau_{v}=-k(v) \mathrm{d} s$.

Let us first analyze the results obtained for the two-component Maxwellian distribution.

Plots of $k / k_{0}$ as a function of $R$ for different values of the ratio $T_{0} / T_{1}$ are shown in Fig. 1 . The $k / k_{0}$ ratio is totally insensitive to the lower temperature $T_{0}$ and, for $T_{0} / T_{1}<0.1$, depends very little also on the temperature ratio. 


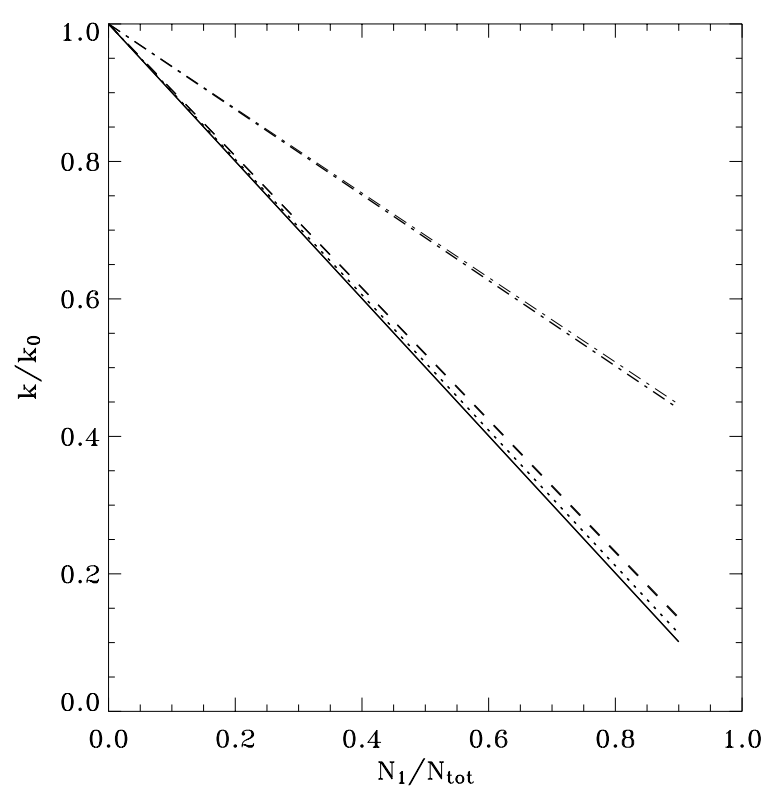

Fig. 1. Ratio of the absorption coefficients $k / k_{0}$ plotted as a function of $R$ for the following values of $T_{0} / T_{1}: 0.5$ (dotted-dashed), 0.1 (dashed), 0.05 (dotted), 0.01 (full). Each curve is plotted for $T_{0}=5 . \times 10^{4}$ and $T_{0}=5 . \times 10^{5}$, but they are not distinguishable.

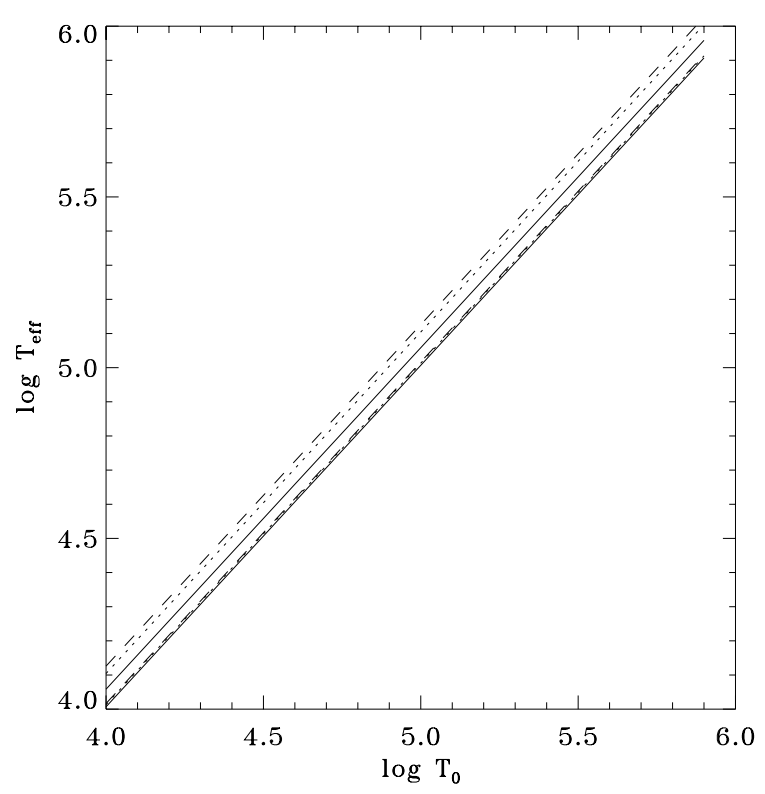

Fig. 2. $T_{\text {eff }}$ vs. $T_{0}$. Lower lines: $R=0.1, T_{0} / T_{1}=0.01$ (full line), 0.05 (dotted), 0.1 (dashed). Upper lines: $R=0.5$, same ratios of temperatures.

The effective temperature is plotted in Fig. 2 as a function of $T_{0}$ for $R=0.1$ and 0.5 and three values of the ratio $T_{0} / T_{1}$. The plot shows that $T_{\text {eff }}$ is strictly proportional to $T_{0}$.

Since both the ratios $k / k_{0}$ and and $T_{\text {eff }} / T_{0}$ are independent of the local temperature $T_{0}$ and depend only on $R$, if we assume that the fraction of particles in the hottest Maxwellian is constant along the TR, we may easily integrate Eq. (13) by multiplying $k_{0}$ and $T_{0}$ by the above ratios.

The brightness temperature $T_{\mathrm{b}}$ for the cell centre and the network are computed using the DEM curves shown by the dotted lines in Fig. 3 of Paper I. They are derived from the VAL

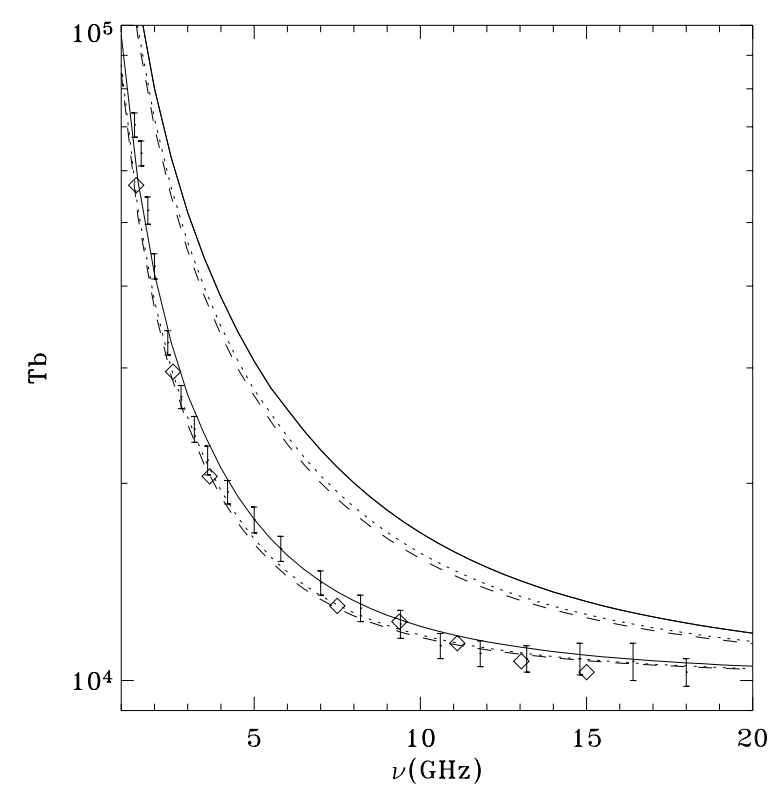

Fig. 3. Brightness temperature spectrum obtained using the TR and coronal models for the cell and the network descibed in the text and assuming, for the electron distribution, a Maxwellian (full lines) and a two-component Maxwellian with $T_{0} / T_{1}=0.1$ and $R=0.2$ and 0.25 (dotted and dashed lines respecively). Points with error bars are from Zirin et al. (1991), diamonds from Borovik et al. (1992).

(Vernazza et al. 1981) model B (cell centre) and D (average network) for $4 \leq \log T \leq 4.3$; from the line intensities observed by SUMER at $\log T \sim 4.5$ and from an interpolation of DEM derived from the line intensities observed by SUMER and CDS at higher temperature. In the SUMER and CDS data analysis the line intensity in the cell center and in network were separately analysed. At $T_{\mathrm{c}}=1.2 \times 10^{6} \mathrm{~K}$ an isothermal corona in hydrostatic equilibrium with a base density $N_{\mathrm{e}}=2 \times 10^{8} \mathrm{~cm}^{-3}$ was assumed. The reason why we used this composite model of the TR is detailed in Paper I. The assumption of an isothermal corona is justified by the fact the most of the coronal contribution comes from the region around the temperature maximum, where the temperature profile is rather flat.

The microwave spectrum has been computed for the cell and the network models in the assumption of thermodynamic equilibrium (full lines in Fig. 3 ). We have then changed $k_{0}$ and $T_{0}$ into $k$ and $T_{\text {eff }}$ obtained assuming $T_{0} / T_{1}=0.1$ and $R=0.2$ and $R=0.25$. The resulting radio $T_{\mathrm{b}}$ are plotted in Fig. 3.

It appears from the figure that the presence of a number of suprathermal particles equal to about $20 \%$ of the total number of electrons decreases the computed $T_{\mathrm{b}}$ from $\sim 10$ to $\sim 2 \%$ going from the lowest to the highest frequencies.

We will now present the results obtained with a population of electrons distributed according to a kappa-function.

In Fig. 4 we have plotted, as a funtion of temperature, the ratios between the absorption coefficients $k_{n} / k_{0}$ and the effectve temperatures $T_{\text {eff }}(n) / T$ for different values of $n$ between $n=3$ and $n=20$.

We see that, also in this case, those ratios do not vary appreciably with temperature and therefore we may compute the 


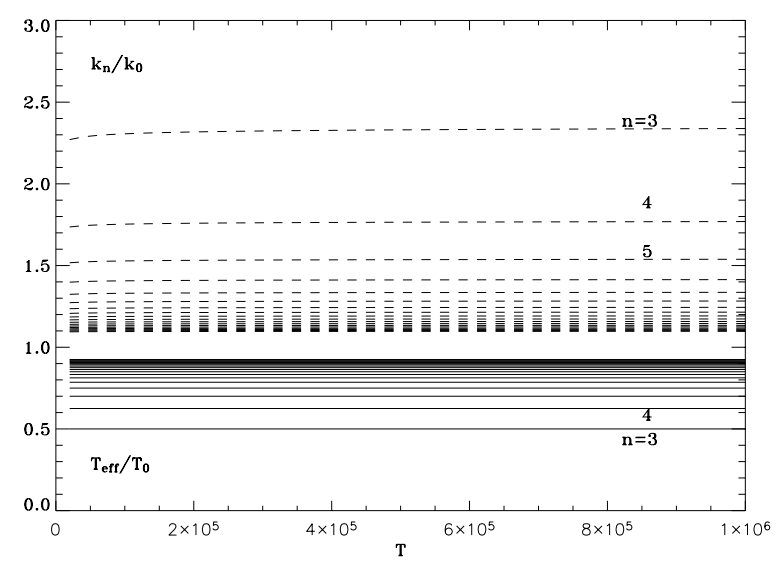

Fig. 4. Ratios of $k_{n} / k_{0}$ (dashed lines) and of $T_{\text {eff }}(n) / T$ (full linws) plotted as a function of T for different values of $n: 3 \leq n \leq 20$.

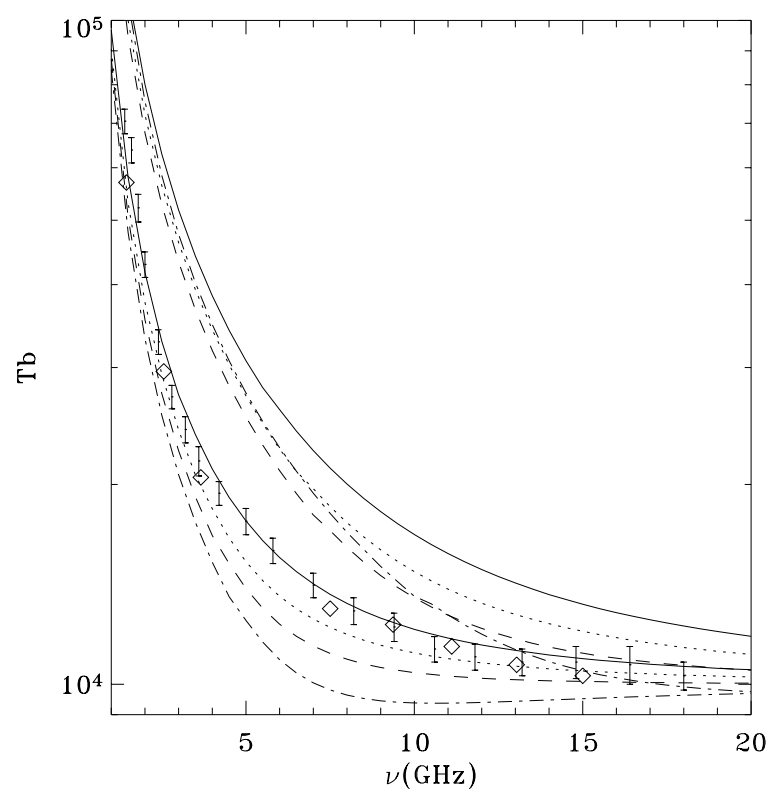

Fig. 5. Microwave spectra of the cell and the network computed using the models mentioned in the text in the assumption of a thermal distribtion of electrons (full lines) and of a population of electrons following a kappa-function with different index: $n=3$ (dot-dashed lines) $n=4$ (dashed), $n=6$ (dotted). Observational points are the same as in Fig. 3.

radio brightness temperature simply by multiplying the temperature and the absorption coefficient of a thermal distribution by the corresponding ratios. This has been done for the network and for the cell center models mentioned above with 4 values of $n: 3 \leq n \leq 6$. The results are shown in Fig. 5 .

It appears from Figs. 3 and 5 that in both cases a distribution of electrons presenting a tail of suprathermal particles has the effect of decreasing the computed $T_{\mathrm{b}}$ in agreement with the expectation of the present calculations.

The way in which this decrease is obtained depends on the details of the distribution function. In case of a two-component Maxwellian, we have an slight increase of the effective temperature, but a decrease of the absorption coefficient, resulting in a decrease of $T_{\mathrm{b}}$. In case of the kappa-function the same effect
Table 1. Fraction of the solar surface covered by cells.

\begin{tabular}{c|c|c|c|c|c}
\hline \hline \multicolumn{2}{c}{ Two-component Maxwellian } & \multicolumn{3}{c}{ Kappa-function } \\
\hline & $R=0.2$ & $R=0.25$ & $n=4$ & $n=5$ & $n=6$ \\
$\alpha$ & 0.95 & 0.9 & 0.75 & 0.8 & 0.83 \\
\hline
\end{tabular}

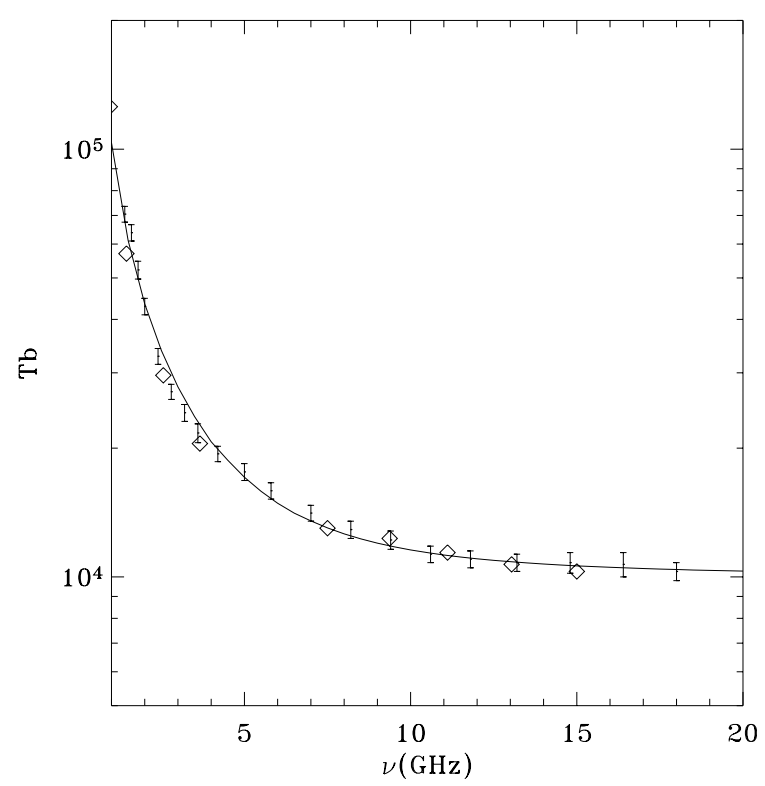

Fig. 6. Computed and observed radio brighteness temperature. The curve is obtained assuming a population of electrons following a kappa-function distribution with index $n=5$ both in the cell and in the network. The assumed fraction of the solar surface covered by the network is $20 \%$.

is produced by a decrease of the effective temperature and an increse of the absorption coefficient.

An inspection of Figs. 3 and 5 indicates that the decrease of $T_{\mathrm{b}}$ in a two-component Maxwellian (unless unacceptable high values of $R$ are assumed) is less evident than that found with the kappa-function, even with index $n \geq 5$, as proposed by Landi \& Pantellini (2001).

If the computed brightness temperature of the cell center decreases below the quiet sun observations, it is possible to add a certain contribution of network emission without exceeding the observations. Calling $\alpha$ the fraction of solar surface covered by cells and $(1-\alpha)$ that covered by the network, the resulting quiet sun brightness temperature is given by:

$T_{\mathrm{b}}(\mathrm{q} . \mathrm{s})=\alpha T_{\mathrm{b}}($ cell $)+(1-\alpha) T_{\mathrm{b}}($ netw $)$.

We have taken all the computed non thermal spectra and found the value of $\alpha$ which best fits the observations. The results are shown in Table 1.

The table shows that with indices of the kappa-function $n=$ 5 and $n=6$, radio observations agree with a network structure covering about $20 \%$ of the solar surface.

Of course the value of $\alpha$ varies with height in the TR, and the given value could be indicative of the low portion of the TR $\left(T \leq 10^{5}\right)$ where most of microwave emission originates. In Fig. 6 the curve obained with $n=5$ and $\alpha=0.8$ is shown. 


\section{Summary and conclusions}

In this paper we have computed the microwave emissivity, absorption coefficient, source function and effective temperature in the assumption that the population of electrons in the solar TR and corona contains a small tail of suprathermal particles. The purpose of this calculation was to check wether this assumption could remove the disagreement between the observed microwave spectra and those computed using UV and EUV based models, which supply values in excess to the former.

The electron population has been modelled

- With two Maxwellians at temperature $T_{1}$ and $T_{0}$ with $T_{0} / T_{1}=0.1$ and with a number of particles, in the hottest one, $N_{1} / N_{\text {tot }} \sim 0.2$.

- With a kappa-function with an integer index $3 \leq n \leq 6$.

A decrease in the computed $T_{\mathrm{b}}$ has been found with both distributions. We do not expect that this result could be modified by changing the form of the electron distribution function. We thus have an indication that the presence of suprathermal particles influences the computed radio brightness in the sense required for a better agreement with the models deduced from EUV observations. Of course, suprathermal electrons also modify the ionization equilibria, because all the ionization and recombination processes are influenced by the form of the distribution functions, as proved e.g. by Dzifčaková (2000). The induced modifications are subtle and a general statement on these matters does not appear to be possible. Therefore, the present study should be complemented by a careful analysis of the effects of suprathermal electrons on UV/EUV computed line intensities. Such a study, that we hope to be able to perform in the near future, is, however outside of the scope of the present paper.

\section{References}

Abramowitz, M., \& Stegun, I. A. 1970, Handbook of Mathematical Functions (New York: Dover)

Borovik, V. N., Kurbanov, M. S., \& Makarov, V. V. 1992, Sv. A., 36, 656B

Dzifčaková, E. 2000, Sol. Phys., 196, 113D

Landi, E., \& Chiuderi Drago, F. 2003, ApJ, 589, 1054 (Paper I)

Landi, S. \& Pantellini, F. G. 2001, A\&A, 372, 686

Ljepojevic, N. N., \& Burgess, A. 1990, Proc. R. Soc. A, 428, 71

Maksimovic, M., Pierrard, V., \& Lemaire, J. F. 1997, A\&A, 324, 725

Maksimovic, M., Pierrard, V., \& Riley, P. 1997, GeoRL, 24, 1151M

Noci, G. 2003, Mem. Soc. Astron. It, 74, 704

Oster, L. 1961, Rev. Mod. Phys., 33, 525

Rohlfs, K., \& Wilson, T. L. 2000, Tools of Radio Astronomy (Berlin: Springer)

Scudder, J. D. 1992, ApJ, 398, 319S

Sheldon, R. B., Adrian, M. L., Chang, S., \& Collier, M. 2001, AGU Spring Meeting 2001, SH 22E-09

Scheuer, P. A. G. 1960, MNRAS, 120, 231

Sittler, E. C. 2002, AGU Spring Meeting 2002, SH 22A-02

Vernazza, J. E., Avrett, E. H., \& Loeser, R. 1981, ApJS, 45, 635

Viñas, A. F., Wong, H. K., \& Klimas, A. J. 2000, ApJ, 528, 509

Wild, J. P., Smerd, S., \& Weiss, A. A. 1963, ARA\&A, 1, 291

Zirin, H., Baumert, B. M., \& Hurford, G. J. 1991, ApJ, 370, 779

Zheleznyakov, V. V. 1970, Radio Emission of the Sun and Planets (Oxford: Pergamon) 


\section{Online Material}




\section{Appendix}

We present here a few details of the calculations leading to the formulas quoted in the main text in connection of the use of kappa-functions as distribution functions.

The emissivity is given by Eq. (2):

$j_{v}=2 \int_{0}^{\infty} \epsilon_{v}(E) F(E) E^{1 / 2} \mathrm{~d} E$.

Taking into account the expression for $\epsilon_{v}(E)$ (Eq. (4)), we see that, apart from the factors independent of $E$, the basic integral for the evaluation of the emissivity is:

$I_{n}=\int_{0}^{\infty} G_{n}(E) \xi(E) \mathrm{d} E$,

where $G_{n}(E)$ has the expression given after Eq. (8) for $k=n$ and $\xi(E)$ is defined as:

$\xi(E)=\ln \left(a E^{3 / 2}\right)-\gamma$

with

$a=\frac{2^{3 / 2}}{\pi Z e^{2} m^{i / 2} v}$.

Assuming as a new variable:

$t=\frac{1}{n-3 / 2} \frac{E}{k_{\mathrm{B}} T}$

we obtain:

$I_{n}=(n-3 / 2)\left(k_{\mathrm{B}} T\right)\left(C_{n} \int_{0}^{\infty} \frac{\mathrm{d} t}{1+t}+\frac{3}{2} \int_{0}^{\infty} \frac{\ln (t)}{(1+t)^{n+1}}\right)$,

where

$C_{n}=\ln \left[(n-3 / 2)^{3 / 2} a\left(k_{\mathrm{B}} T\right)^{3 / 2}\right]-\gamma$

or, using Eq. (5):

$C_{n}=1 / 2(\Lambda+3[\ln (n-3 / 2)+\gamma])$.

The integrals entering the expression for $I_{n}$ can be integrated explicitly giving:

$\int_{0}^{\infty} \frac{\mathrm{d} t}{(1+t)^{n+1}}=\frac{1}{n}$

$\int_{0}^{\infty} \frac{\ln (t)}{(1+t)^{n+1}} \mathrm{~d} t=\frac{1}{n} \sum_{k=1}^{n-1} \frac{1}{k}$

Therefore

$$
\begin{aligned}
I_{n} & =\frac{n-3 / 2}{2 n} k_{\mathrm{B}} T\left(\Lambda+3\left[\ln (n-3 / 2)+\gamma-\sum_{k=1}^{n-1} \frac{1}{k}\right]\right) \\
& =\frac{n-3 / 2}{2 n} k_{\mathrm{B}} T[\Lambda+3(\Omega+1 / n)],
\end{aligned}
$$

where

$\Omega=\ln (n-3 / 2)+\gamma-\sum_{k=1}^{n} \frac{1}{k}$.
Taking into account the constant factors so far neglected, we finally arrive at:

$j_{n}(v)=\frac{2 C}{\sqrt{\pi k_{\mathrm{B}}}} \frac{N_{i} N_{\mathrm{e}}}{T^{1 / 2}} \frac{n-3 / 2}{n} H_{n}[\Lambda+3(\Omega+1 / n)]$.

Using Stirling's asymptotic formula for the Gamma function it is easily seen that:

$\lim _{n \rightarrow \infty} H_{n}=1$,

and that (Abramowitz et al. 1970)

$\lim _{n \rightarrow \infty} \Omega=\lim _{n \rightarrow \infty}\left(\gamma-\sum_{k=1}^{n} \frac{1}{k}+\ln (n)\right)=0$,

so that $j_{n}(v)$ converges to $j_{0}(v)$ when $n \rightarrow \infty$.

Turning now to the absorption coefficient (Eq. (3)):

$k_{v}=-\frac{c^{2}}{v^{2}} \int_{0}^{\infty} \frac{\mathrm{d} F(E)}{\mathrm{d} E} \epsilon_{v}(E) E^{1 / 2} \mathrm{~d} E$,

and taking into account the form of $\epsilon_{v}(E)$ we see that the basic integral to be evaluated for the computation of $k_{v}$ is:

$K_{n}=\int_{0}^{\infty} \frac{\mathrm{d} G_{n}(E)}{\mathrm{d} E} \xi(E) \mathrm{d} E$.

Performing the derivative and introducing again the variable $t$, we see that:

$K_{n}=-(n+1)\left(C_{n} \int_{0}^{\infty} \frac{\mathrm{d} t}{(1+t)^{n+2}}+\frac{3}{2} \int_{0}^{\infty} \frac{\ln (t)}{(1+t)^{n+2}}\right)$.

Proceeding as before we arrive at:

$$
\begin{aligned}
K_{n} & =-(n+1)\left(C_{n} \frac{1}{n+1}-\frac{3}{2} \frac{1}{n+1} \sum_{k=1}^{n} \frac{1}{k}\right) \\
& =\frac{1}{2}(\Lambda+3 \Omega) .
\end{aligned}
$$

The final expression for $k_{v}$ turns out to be:

$k_{n}(v)=\frac{2 C}{\sqrt{\pi k_{\mathrm{B}}}} \frac{c^{2}}{2 k_{\mathrm{B}} T v^{2}} \frac{N_{i} N_{\mathrm{e}}}{T^{1 / 2}} H_{n}(\Lambda+3 \Omega)$. 